\title{
The persistence length of double stranded DNA determined using dark field tethered particle motion
}

\author{
Sanneke Brinkers, ${ }^{1, a)}$ Heidelinde R. C. Dietrich, ${ }^{1}$ Frederik H. de Groote,${ }^{2}$ Ian T. Young, ${ }^{1}$ \\ and Bernd Rieger ${ }^{1}$ \\ ${ }^{1}$ Quantitative Imaging Group, Faculty of Applied Sciences, Delft University of Technology, \\ Lorentzweg 1, 2628 CJ Delft, The Netherlands \\ ${ }^{2}$ Leiden Institute of Chemistry, Leiden University, Einsteinweg 55, 2333 CC Leiden, The Netherlands
}

(Received 23 December 2008; accepted 2 May 2009; published online 4 June 2009)

\begin{abstract}
The wormlike chain model describes the micromechanics of semiflexible polymers by introducing the persistence length. We propose a method of measuring the persistence length of DNA in a controllable near-native environment. Using a dark field microscope, the projected positions of a gold nanoparticle undergoing constrained Brownian motion are captured. The nanoparticle is tethered to a substrate using a single double stranded DNA (dsDNA) molecule and immersed in buffer. No force is exerted on the DNA. We carried out Monte Carlo simulations of the experiment, which give insight into the micromechanics of the DNA and can be used to interpret the motion of the nanoparticle. Our simulations and experiments demonstrate that, unlike other similar experiments, the use of nanometer instead of micrometer sized particles causes particle-substrate and particle-DNA interactions to be of negligible effect on the position distribution of the particle. We also show that the persistence length of the tethering DNA can be estimated with a statistical error of $2 \mathrm{~nm}$, by comparing the statistics of the projected position distribution of the nanoparticle to the Monte Carlo simulations. The persistence lengths of 45 single molecules of four different lengths of dsDNA were measured under the same environmental conditions at high salt concentration. The persistence lengths we found had a mean value of $35 \mathrm{~nm}$ (standard error of $2.8 \mathrm{~nm}$ ), which compares well to previously found values using similar salt concentrations. Our method can be used to directly study the effect of the environmental conditions (e.g., buffer and temperature) on the persistence length. (C) 2009 American Institute of Physics.
\end{abstract}

[DOI: $10.1063 / 1.3142699$ ]

\section{INTRODUCTION}

Research into the properties of polymers such as double stranded DNA (dsDNA) has made a transition from bulk experiments to single-molecule experiments. Bulk experiments provide results averaged over the population, while single-molecule methods provide a clearer understanding of the mechanics of individual molecules. The micromechanics of dsDNA can be described by several models, the best known of which is the wormlike chain (WLC) model $^{1}$ for semiflexible polymers. It describes dsDNA especially well if the forces exerted on the DNA are small $(<10 \mathrm{pN})^{2}$

The WLC model describes the conformations of the polymer as a curve with a certain correlation length in the direction along the contour. This correlation length is called the persistence length of the polymer and is the basis of the entropic elasticity of semiflexible polymers. Many singlemolecule experiments have been carried out to determine the persistence length of dsDNA. Optical ${ }^{3,4}$ or magnetic tweezers ${ }^{5-7}$ have been used to apply a force to the DNA and obtain a force-extension curve. Fitting the WLC model to such a curve provides the persistence length of the dsDNA. ${ }^{8}$ Other well-known methods include depositing the DNA onto a substrate and imaging its shape using either atomic force

${ }^{a)}$ Electronic mail: s.brinkers@tudelft.nl. microscopy ${ }^{9-11}$ (AFM) or electron microscopy (EM). ${ }^{12,13}$ In the images collected using AFM or EM, the contours of the DNA are traced from which the persistence length can be determined.

In this article, we describe a method for measuring the persistence length of dsDNA in a controllable environment. No force is exerted on the dsDNA and the molecules are not confined to a two-dimensional (2D) surface, therefore they can adopt more natural three-dimensional (3D) conformations. We use tethered particle motion (TPM), where a small reporter particle is tethered to a substrate using a single dsDNA molecule. The particle-molecule system is allowed to exhibit (confined) Brownian motion. The particle's motion is influenced by the (micro-) mechanical properties of the tethering molecule and the environmental conditions (see Fig. 1). By following the motion of the particle, properties of the tether can be deduced. Using TPM the influence of different buffers and temperatures on the mechanical properties of dsDNA can be examined without applying any external forces on the DNA.

TPM has been used for a number of applications, including studying DNA-protein interactions, ${ }^{14,15}$ DNA and RNA transcription, ${ }^{16,17}$ looping and supercoiling of DNA, ${ }^{18-20}$ and the determination of mechanical properties of DNA/RNA. ${ }^{21-23}$ In these cases (except Ref. 21) the reporter particle is a micrometer-sized polystyrene particle. The large 


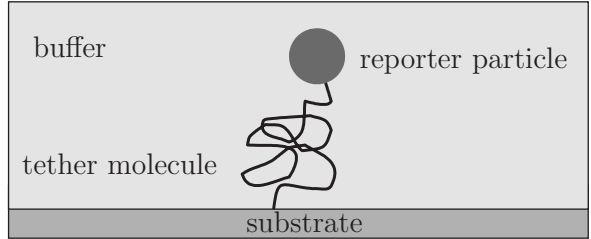

FIG. 1. Principle of tethered particle motion: A chain molecule is used to tether a reporter particle to a substrate. The reporter particle exhibits Brownian motion influenced by the mechanical properties of the tether.

size of the reporter particle compared to the DNA (lengths on the order of 100-2000 nm are generally used) causes the position distribution of the reporter particle to differ from the Gaussian distribution that is characteristic of Brownian motion. According to Segall et $a .^{24}$ this is to be attributed to a volume exclusion effect due to steric hindrance of the particle near the substrate.

In contrast to the above mentioned methods, we use nanometer-sized gold particles (diameter of $80 \mathrm{~nm}$ ). In our case the dimensions of the particle are small enough such that volume exclusion effects caused by the particle's proximity to the substrate do not influence the motion.

These highly scattering gold nanoparticles ${ }^{25}$ are made visible using an (objective-type) dark field microscope and imaged using a charge coupled device (CCD) camera. This combination (dark field TPM) produces images with high contrast and high signal-to-noise ratio, using a relatively simple setup. ${ }^{21}$ The persistence length of the tethering dsDNA can be determined by statistically comparing the position distribution of the nanoparticle to computer simulations of the experiment where the dsDNA is modeled by the wormlike chain model.

\section{THEORETICAL BACKGROUND}

\section{A. Wormlike chain model}

In the WLC model, ${ }^{1}$ semiflexible polymers are described by their bending rigidity. On short length scales it takes considerable energy to bend the polymers, while on longer length scales the molecule can be bent and curved much more easily. The characteristic bending length scale is called the persistence length. The persistence length $(P)$ is mathematically defined as the decay length of tangent-tangent correlations of the polymer:

$$
\left\langle\vec{t}(s) \cdot \vec{t}\left(s^{\prime}\right)\right\rangle=e^{-\left|s-s^{\prime}\right| / P} .
$$

Here $\vec{t}(s)$ and $\vec{t}\left(s^{\prime}\right)$ are tangents to the polymer at two points a distance $\left|s-s^{\prime}\right|$ apart on the contour of the polymer (see Fig. 2). The energy needed to bend a semiflexible polymer over an angle $\theta$ over a length $l$ depends on the temperature $T$ and the persistence length $P$ of the polymer:

$$
E_{\mathrm{WLC}}=\frac{k_{B} T P}{2 l} \theta^{2} .
$$

Thermal fluctuations give rise to an entropic elasticity with an effective spring constant given in Eq. (3) for a dsDNA molecule of contour length $L$ (approximation for small forces): ${ }^{26}$

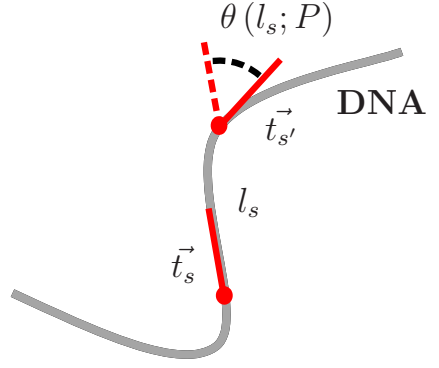

FIG. 2. (Color online) Bending a semiflexible polymer over an angle $\theta$ between two tangents $\left(t_{1}\right.$ and $\left.t_{2}\right)$ a distance $l$ over the contour apart.

$$
k_{\mathrm{WLC}}=\frac{3 k_{B} T}{2 P L} .
$$

The central moments of the end-to-end distance $(R)$ distribution [see Eq. (4)] have been analytically determined. ${ }^{27}$ The second central moment for $2 \mathrm{D}$ and $3 \mathrm{D}$ conformations is the same, however the $2 \mathrm{D}$ persistence length is twice the $3 \mathrm{D}$ persistence length:

$$
\begin{aligned}
& \langle R\rangle=0, \\
& \left\langle R_{3 \mathrm{D}}^{2}\right\rangle=2 P_{3 \mathrm{D}} L-2 P_{3 \mathrm{D}}^{2}\left(1-e^{-L / P_{3 \mathrm{D}}}\right), \\
& \left\langle R_{2 \mathrm{D}}^{2}\right\rangle=2 P_{2 \mathrm{D}} L-2 P_{2 \mathrm{D}}^{2}\left(1-e^{\left.-L / P_{2 \mathrm{D}}\right),}\right. \\
& P_{2 \mathrm{D}}=2 P_{3 \mathrm{D}} .
\end{aligned}
$$

Considerable effort has been made to analytically derive the complete end-to-end distance distribution of WLCs. ${ }^{28-31}$ Since in TPM experiments the dsDNA molecule is attached to a substrate at one end and the other end carries a reporter particle, the influence of these factors has to be taken into account. Although analytical approaches were used to determine the distribution for WLCs with constrained ends, ${ }^{29,30}$ as of yet there is no simple analytical formula for the position distribution of tethered particles. We therefore take a different approach: We obtain the position distribution by sampling a large number of possible dsDNA and particle conformations using Monte Carlo simulations and determine the simulated position distribution of the particle.

\section{B. Volume exclusion effect}

In our TPM experiments the observable is the (projected) motion of a particle attached to the free end of the DNA. Directly fitting the WLC model to our observations disregards the influence of interactions between the substrate, DNA, and reporter particle. The influence of excluded volume by particle-substrate interactions was analyzed in a recent article by Segall et al. ${ }^{24}$ The particle-substrate interaction is modeled as a hard-wall interaction, which limits the conformations that the DNA can adopt and results in an effective stretching force on the DNA. Segall et al. define a dimensionless number, the excursion number $N_{R}$, as a function of the particle radius $R_{p}$ and the contour length $L$ and persistence length $P$ of the polymer chain: 


$$
N_{R}=\frac{R_{p}}{\sqrt{P L / 3}} .
$$

For situations where the excursion number is larger than 1, the volume exclusion effect is such that the DNA is effectively stretched. The Brownian motion of the particle is said to be "particle dominated." At excursion numbers $<1$ the motion is not significantly influenced by the particle and therefore the motion is "molecule-dominated." Segall et al. go on to show the influence of the excursion number on the central moments of a freely jointed chain (FJC), which is a more general model for flexible polymers. The end-to-end distance distribution of a freely jointed chain is Gaussian with a mean square end-to-end distance $\left\langle R_{3 \mathrm{D}}^{2}\right\rangle=2 P L,{ }^{32}$ equal to the WLC model for polymers with a long contour length $(L \gg P)$. The influence of the excursion number on the second central moments of the end-to-end distance for freely jointed chains is given in Eq. (6) [see Ref. 24, Eq. (10)]:

$$
\begin{aligned}
& \left\langle R_{\perp}^{2}\right\rangle=\frac{P L}{3}\left(2+\frac{4 N_{R}}{\sqrt{\pi} \operatorname{erf}\left(N_{R}\right)}\right), \\
& \left\langle R_{z}^{2}\right\rangle=\frac{P L}{3}\left(2+\frac{4 N_{R}}{\sqrt{\pi} \operatorname{erf}\left(N_{R}\right)}+N_{R}^{2}\right) .
\end{aligned}
$$

Here $\left\langle R_{\perp}^{2}\right\rangle$ and $\left\langle R_{z}^{2}\right\rangle$ are the second central moments of the projected radius and height of the position of the particle, respectively. Adding the height and projected radius (which can be done as they are independent of each other) gives the following equation for the 3D mean square end-to-end distance:

$$
\left\langle R_{3 \mathrm{D}}^{2}\right\rangle=\frac{P L}{3}\left(4+\frac{8 N_{R}}{\sqrt{\pi} \operatorname{erf}\left(N_{R}\right)}+N_{R}^{2}\right) .
$$

In the limit of $N_{R} \searrow 0$ Eqs. (6) and (7) give the same results as the FJC model without adaptations.

\section{EXPERIMENTAL METHODS}

\section{A. Monte Carlo simulations}

Monte Carlo simulations were carried out to determine the position distribution of the particles in TPM experiments. The WLC model was used to incorporate the mechanical properties of the tethering dsDNA. Apart from simulations of TPM experiments, we also simulated the end-to-end distance distribution of free DNA molecules, as well as the position distributions of the free end of DNA molecules attached to a substrate at one end. The simulations were carried out in MATLAB and included the influence of the physical properties of the substrate and nanoparticle. Our implementation is based on an article and (MATHEMATICA) code provided by Nelson et al. ${ }^{22}$

For each conformation, the DNA molecule is built as a chain of segments with length $l_{s} \ll P$. Each segment has a certain (bending) angle with respect to the previous segment, which is randomly drawn from the statistical distribution of the bend angle given in the WLC model. This distribution is obtained from Boltzmann statistics of the energy needed for a certain bending angle [Eq. (2)]: ${ }^{11}$

$$
\operatorname{Pr}\left(\theta \mid l_{s}, P\right)=\sqrt{\frac{P}{2 \pi l_{s}}} e^{-P \theta^{2} / 2 l_{s}} .
$$

In TPM experiments the DNA is attached to a substrate using a freely pivoting connection and we simulate it as such. The interactions of the DNA and nanoparticle with the substrate are modeled as hard-wall interactions. This is implemented in the simulations by taking the orientation of the first segment confined to the upper half-space $(z>0)$ and discarding any configurations where the chain or nanoparticle crosses the $z=0$ plane. The nanoparticle attached at the free end of the DNA is freely pivoting around the end of the DNA. For each DNA configuration, the orientation of the nanoparticle is randomly drawn from a uniform distribution. If the nanoparticle intersects with the DNA in any segment or if it intersects with the substrate the conformation is discarded.

The simulations are carried out for 81 different persistence lengths from 10 to $90 \mathrm{~nm}$ in steps of $1 \mathrm{~nm}$. For each persistence length conformations are generated until a total of $10^{5}$ allowed conformations have been obtained. This is done for each of the following three cases: (1) free molecules, (2) molecules attached to a substrate at one end, and (3) molecules attached to a substrate at one end and a reporter particle bound at the other end, as in our TPM experiments. Generating $10^{5}$ allowed conformations for a $480 \mathrm{~nm}$ long DNA molecule with a persistence length of $50 \mathrm{~nm}$ takes approximately half an hour on a modern PC.

\section{B. TPM experiments}

TPM experiments (as opposed to simulations) were carried out on double stranded DNA of four different contour lengths: 123, 482, 908, and $1660 \mathrm{~nm}(362,1417,2672$, and 4882 base pairs, respectively). The DNA is coupled at one end to a gold substrate using thiol-thiol binding. At the other end a gold nanoparticle (diameter of $80 \mathrm{~nm}$ ) is bound using biotin-antibiotin binding. The different lengths are all observed in the same environmental conditions (buffer, temperature). The excursion numbers as defined in Eq. (5) for the different DNA lengths are all below one $(0.88,0.45,0.33$, and 0.24 for the different contour lengths, respectively, using a value of $50 \mathrm{~nm}$ for the persistence length). According to Segall et al. the motion of the nanoparticle is therefore not dominated by its proximity to the surface and there is no effective force stretching the DNA.

\section{1. dsDNA fragment preparation}

The dsDNA fragments were synthesized from an unmethylated lambda DNA (Promega, NL) template using Expand Long Template PCR System (Roche, NL). dNTPs were bought from Promega, NL. The following primers (Isogen, NL) were used for the production of all fragments: 5'-biotin-ATA GGC CAG TCA ACC AGC AGG-3' (forward), 5' -disulfide-ATA GGT AAA GCG CCA CGC TCC-3' (reverse), 5'-disulfide-GGG ATA ATC GGC GTG GCA GAT AAC-3' (reverse, for 2672 bp fragments only). 5'-disulfide-GCA GCT TCT GAC CGC AGT TAG CG-3' (reverse, for 1417 bp fragments only), 5'-disulfide-TCC 
AAG CTC CGG GTT GAT ATC AAC C-3' (reverse, for 362 bp fragments only). The PCR product was purified according to the QIAquick PCR purification kit manual (Qiagen, NL).

\section{TPM sample preparation}

All steps in the preparation of the TPM chips were carried out in a humidified surrounding. Gold supports (Arrandee, GER) were incubated with $1 \mathrm{mM}$ hexanethiol (Fluka, NL) diluted in toluene (Sigma, NL) for approximately 60 min and subsequently washed with toluene and isopropanol for at least $30 \mathrm{~min}$ each. This step prevents the nonspecific binding of DNA to the gold surface. The hexanethiol chips were divided into four sections, one for each of the different DNA fragments. Onto each section $0.5 \mathrm{mg} / \mathrm{ml}$ biotin in TE buffer $\left(10 \mathrm{mM}\right.$ tris-HCl, $1 \mathrm{mM}$ EDTA; $p \mathrm{H} 8.0$ at $\left.21{ }^{\circ} \mathrm{C}\right)$ was applied for $15 \mathrm{~min}$ and subsequently washed with TE buffer. The immobilized biotin allowed the production of stationary beads as the gold nanoparticles are coated with antibiotin antibodies. For each length, the purified dsDNA was diluted to a concentration of approximately $10 \mathrm{ng} / \mu \mathrm{l}$ in TE buffer, applied onto a separate section of the support and incubated over night at $4{ }^{\circ} \mathrm{C}$. Unbound material was washed away by thorough rinsing with TE buffer. Gold nanoparticles coated with antibiotin antibodies $(80 \mathrm{~nm}$ diameter, British Biocell, U.K.) in TE buffer were applied onto the chip surface and incubated for $60 \mathrm{~min}$. Unbound particles were washed off with TE buffer. The prepared gold slide was kept wet by TE buffer until usage, then it was placed on the lower part of a flow chamber, which was subsequently closed by a cover slip and filled with measurement buffer resembling physiological conditions $(25 \mathrm{mM}$ tris- $\mathrm{HCl}, 100 \mathrm{mM} \mathrm{NaCl} ; p \mathrm{H} \mathrm{7.4)}$. This allowed observation of the sample over several hours.

\section{TPM measurement}

The flow chamber was placed on the specimen holder of an upright microscope (Olympus, NL). A dark field objective (UMplan Fl BD 50x 0.80, Olympus) was used to image the nanoparticles on a frame transfer CCD camera (Hamamatsu C8800). In a dark field objective, the illumination and imaging light paths are separated from each other. The sample is illuminated with a hollow cone of light under an oblique angle. Only scattered light can re-enter the imaging part of the objective, therefore objects that scatter light brightly are imaged against a dark background. Fields of view with several moving and stationary nanoparticles (the latter are used for drift correction) were chosen and 1500-2000 consecutive frames were captured for each field of view. The exposure was set to $10 \mathrm{~ms} /$ frame to collect enough photons for high signal-to-noise ratio images without a significant underestimation of the motion due to motion blur. ${ }^{33}$ A short explanation of the motion blur effect is given in the supplementary material. ${ }^{34}$ The sampling distance in the $x$ and $y$ was $\Delta x$ $=\Delta y=160 \mathrm{~nm}$. The image of an $80 \mathrm{~nm}$ gold nanoparticle is given by the point-spread function (PSF) of the microscope, which can be approximated by a Gaussian. ${ }^{35}$ Figure 3 shows a typical image of a gold nanoparticle attached to DNA, the

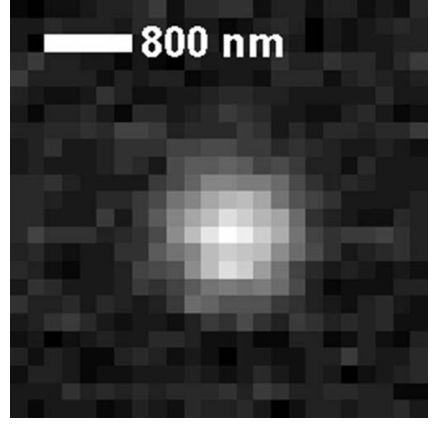

FIG. 3. The dark field image of a gold nanoparticle (SNR is $33 \mathrm{~dB}$ ).

peak signal to noise ratio of which is $33 \mathrm{~dB} .^{43}$ The measured image of the nanoparticle is larger than the theoretical PSF due to imaging through $500 \mu \mathrm{m}$ of buffer.

\section{Analysis}

The position distribution of the tethered nanoparticles was obtained by analyzing the image series. They were analyzed using in-house software based on the MATLAB toolbox DIPimage (Scientific Image processing toolbox, www.diplib.org, Delft University of Technology, Delft, NL). The user manually selects the stationary and moving nanoparticles in the first frame of the image sequence via a graphical user interface. The remaining procedure is fully automated. The positions of all selected nanoparticles are tracked with subpixel precision using a maximum likelihood estimation of the position of the Gaussian image profile. Based on the relative averaged motion of the nanoparticles marked as stationary, the global drift is computed and subtracted from the motion of the moving nanoparticles. No rotation of the sample plane during the experiments has been found. Care has to be taken to only include correctly (singly) tethered particles in the subsequent analysis steps. Multiply tethered particles are identified by asymmetry of the position distribution. ${ }^{19,23}$ For this reason we visually inspected 2D histograms [see, e.g., Fig. 7(a)] of the positions of the moving nanoparticles to verify an isotropic distribution. Blumberg et al. ${ }^{19}$ and Pouget et al. ${ }^{23}$ discarded any position distribution with an anisotropy above $20 \%$ and $10 \%$, respectively. Visual inspection shows that an anisotropy of $10 \%$ is already clearly visible by eye, therefore we can safely assume that we kept only singly tethered particles.

\section{Localization precision}

The localization precision of our method was extensively tested. The theoretical limit to the localization precision of Gaussian image profiles is $\sqrt{\left\langle\Delta x^{2}\right\rangle}=\sigma_{g} / \sqrt{\gamma A t}$, where $\sigma_{g}$ denotes the size of the Gaussian profile and $\gamma A t$ the number of photons collected per particle. ${ }^{35}$ We compared the localization precision of simulated Gaussian blobs to this limit, as well as the remaining detected motion of the stationary particles. Figure 4 shows some results. The localization algorithm has a precision comparable to the theoretical prediction in the absence of (Gaussian) read noise in the camera (data not shown). However, the CCD camera has considerable read noise $\left(30 e^{-} \mathrm{rms}\right)$, therefore Gaussian noise was 


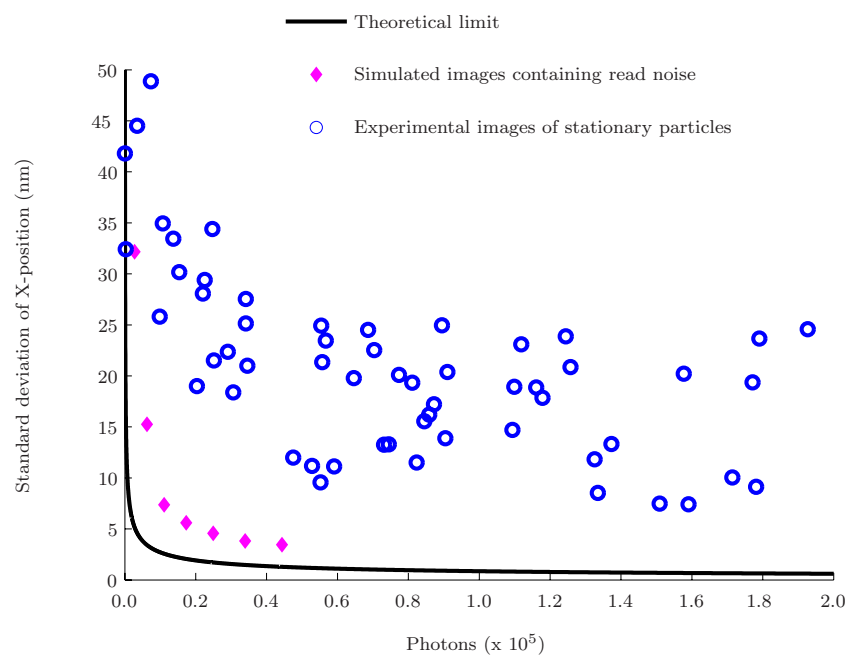

FIG. 4. (Color online) Localization precision for Gaussian image profiles. The solid line represents the theoretical limit by Ober et al. (Ref. 35). The diamond scatter plot is the precision obtained when analyzing simulated images with the same properties as experimentally obtained images. The circular scatter plots represent measurements of the position of nine different stationary particles in TPM experiments. The difference between the simulated images and stationary particles is due to instrument vibrations and residual motion of the particles. The resulting localization precision for our experimental conditions is $30 \mathrm{~nm}$.

added to the simulated images resulting in a more realistic simulation and producing a slightly inferior localization precision. The detected remaining motion of stationary particles is larger than the localization precision as predicted by the simulated images. This indicates the presence of instrument vibrations or residual motion of the stationary particles. In our experiments we used an exposure time of $10 \mathrm{~ms}$, which typically resulted in $(0.1-0.2) \times 10^{5}$ detected photons per bead per image. In that case the standard deviation of the $x$-position of the stationary particles was only on the order of $30 \mathrm{~nm}$. This represents a minor influence on the obtained position distribution for the moving particles. Increasing the exposure time would result in a slightly better precision $(25$ $\mathrm{nm})$, but would lead to a much larger underestimation of the distribution due to motion blur averaging of the positions. ${ }^{33,34}$

\section{Persistence length}

Our method for determining the persistence length of the dsDNA makes use of statistically comparing the measured position distribution to the simulated ones. The simulations were carried out with different persistence lengths ranging from 10 to $90 \mathrm{~nm}$. The statistical test we use is the nonparametric two-sample Kolmogorov-Smirnov (KS) test. ${ }^{36}$ This test compares the cumulative distribution functions $(\mathrm{CDFs})$ of the two distributions $\left(F_{n_{1}}\right.$ and $\left.F_{n_{2}}\right)$. The test statistic $D$ is the maximum vertical distance between the two CDFs:

$$
D=\max _{x}\left|F_{n_{1}}-F_{n_{2}}\right| \cdot
$$

The probability of finding a certain $D$ with sample size $N$ is seen here as the statistical agreement between the measurement and simulation. A large value of $D$, taking into account sample size, leads to a low probability that the measurement

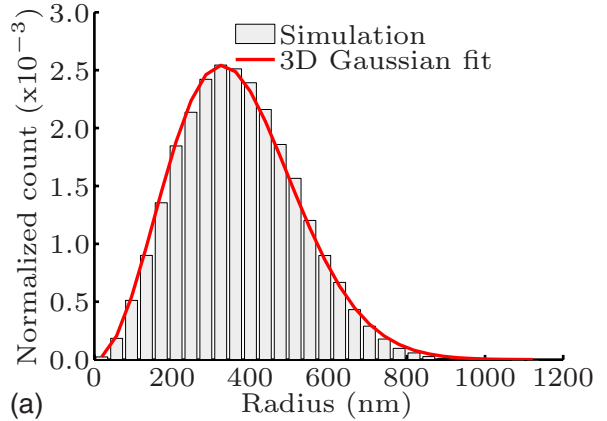

(a)
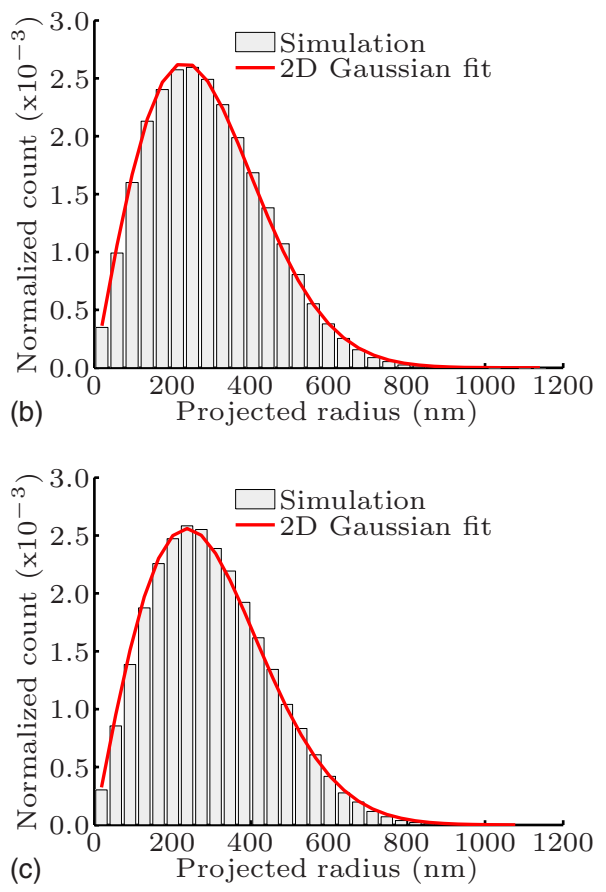

FIG. 5. (Color online) The distribution of the (projected position) of the end point of the dsDNA [Figs. 5(a) and 5(b)] or the center of the nanoparticle [Fig. 5(c)] for simulated dsDNA configurations. Parameters: $L=1660 \mathrm{~nm}$, $P=50 \mathrm{~nm}, R_{p}=40 \mathrm{~nm}$.

of the stochastic process and the simulation of the process are in agreement. The persistence length of the dsDNA is determined by looking for the simulation with which the measured distribution has the highest statistical agreement. The persistence length used in that simulation is the measured persistence length.

\section{RESULTS}

\section{A. Monte Carlo simulations}

In Fig. 5 the results are shown for simulations of dsDNA molecules with a contour length of $4882 \mathrm{bp}(\sim 1660 \mathrm{~nm})$, persistence length of $50 \mathrm{~nm}$, and (where applicable) particle radius of $40 \mathrm{~nm}$. In this case the excursion number is $N_{R}$ $=0.24$. The resulting position distributions for the three different cases (free molecules, molecules attached to a substrate and molecules in TPM experiments) are shown. The histogram in Fig. 5(a) represents the distribution of the endto-end distance of simulated free molecules. Figures 5(b) and 5 (c) show the distribution of the projected radius $\left(R_{\perp}\right.$ $=\sqrt{x^{2}+y^{2}}$ ) of the end point of the DNA and of the center of the attached nanoparticle, respectively. We fitted the distribu- 

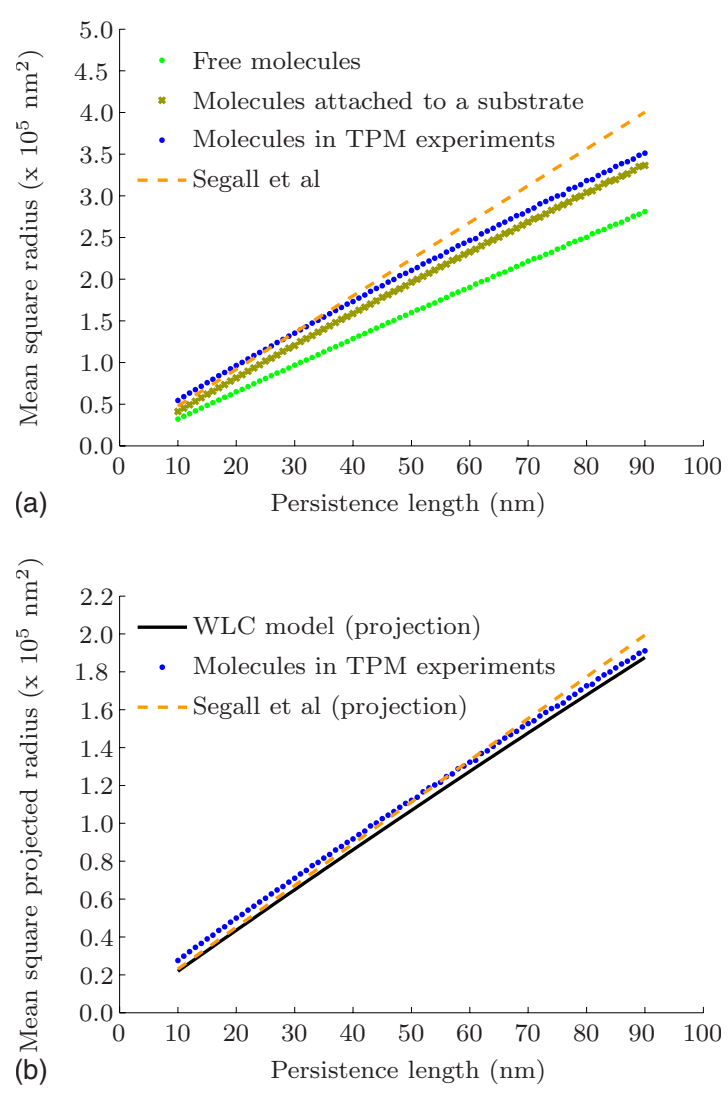

FIG. 6. (Color online) Central moments of WLCs. (a) shows the mean square radius of $10^{5}$ simulated free molecules, molecules attached to a substrate and molecules in TPM experiments (dsDNA length: $4882 \mathrm{bp}$ or 1660 $\mathrm{nm}$, radius of the nanoparticle: $40 \mathrm{~nm}$ ). The variation on each data point is less than $1.5 \%$, therefore no error bars are plotted. The dashed line is the model proposed by Segall et al. In (b) the simulations of molecules in TPM experiments are compared to the WLC model and Segall et al. using only the projected position. The differences between the models and simulation are much less pronounced in that case.

tions with Gaussian distributions (solid lines) since for long molecules $(L \gg P)$ the DNA should have a Gaussian position distribution according to both the FJC and WLC models (central limit theorem). The Gaussian distribution of the radius $r$ in 2D and 3D is given in equation (10): ${ }^{37}$

$$
\begin{aligned}
& \operatorname{Pr}(r, \sigma, 2 \mathrm{D})=\frac{r}{\sigma^{2}} e^{-r^{2} / 2 \sigma^{2}}, \text { for } r>0, \\
& \operatorname{Pr}(r, \sigma, 3 \mathrm{D})=\frac{1}{\sigma} \sqrt{\frac{2}{\pi} \frac{r^{2}}{\sigma^{2}} e^{-r^{2} / 2 \sigma^{2}}, \quad \text { for } r>0 .}
\end{aligned}
$$

The fits show that our simulated distributions follow Gaussian distributions. If the particle-substrate interactions would have caused a significant volume exclusion effect, the distributions would not be Gaussian. This implies that the volume exclusion effect is negligible in our case, as predicted by the small excursion number.

In Fig. 6 an overview of the central moments of the different simulations is given. The mean square radius is plotted as a function of the persistence length used in the simulations. In Fig. 6(a) we show the (3D) mean square endto-end radius of free molecules and molecules attached to a substrate as well as the mean square radius of the position of the particle attached to molecules in TPM experiments. The DNA length used here is $4882 \mathrm{bp}(\sim 1660 \mathrm{~nm})$. Each data point represents the mean square radius of $10^{5}$ conformations. The variation between 20 simulations of 5000 conformations each is less than $1.5 \%$ for each data point, therefore no error bars are presented.

As can be seen in this figure, attaching the molecules to a substrate causes a scaling of the mean square radius compared to free molecules. This scaling is caused by an almost doubling (1.7 times) of the mean square height of the free end of molecules attached to a substrate compared to free molecules. For a freely jointed chain, DiMarzio ${ }^{38}$ derived theoretically that the mean square height doubles when the chain is confined to a half-space. The fact that we find a factor of 1.7 might be due to the differences between the WLC model and the FJC model.

Attaching a nanoparticle to the free end of the molecules results in an additional offset of the mean square radius. The offset represents interactions of the nanoparticle with the DNA and the substrate, however the influence is much less pronounced than the model proposed by Segall et al. [Eq. (6)] which is based on the scaling of the central moments with a dimensionless excursion number. Our simulations do not show the same scaling behavior as Segall et al. predict. In their article, however, they show that the model complies well with their own Monte Carlo simulations. These different behaviors can be explained by the fact that our nanoparticles are of a size that is on the order of the persistence length (diameter of $80 \mathrm{~nm}$ ). Effectively, the DNA molecule will not "feel" an extra segment that is on the same length scale as its own stiffness. We conclude that the scaling model proposed by Segall et al. does not hold in the limit of small particle sizes. $^{39}$

In Fig. 6(b) the mean square radius of the $(x, y)$-projection $\left(\left\langle R_{\perp}^{2}\right\rangle\right)$ is plotted against the persistence length. In this figure we see that the differences between the WLC model (without taking into account the substrate and nanoparticle), the model proposed by Segall et al. and our simulations hardly differ within the measurement uncertainty if only the projected radius (2D) is used.

\section{B. TPM experiments}

Figure 7 shows some of the results for a nanoparticle tethered using a 4882 bp long dsDNA molecule. A 2D histogram of the positions of that nanoparticle is shown in Fig. 7(a). In Fig. 7(b) a histogram of the projected (radial) position is shown. The line represents a fit of a 2D Gaussian distribution to our data. The goodness of the fit denotes that our experiments too are not restricted by nanoparticlesubstrate interactions. In Fig. 8 the results for the two-sample KS tests with the simulations with different persistence lengths is shown. The measured persistence length here is 50 $\mathrm{nm}$ with an error [full width at half maximum (FWHM) of the probability peak] of $2.0 \mathrm{~nm}$.

In total 45 nanoparticles showing an isotropic position distribution were found for the four dsDNA lengths (6 for $123 \mathrm{~nm}, 18$ for $482 \mathrm{~nm}, 12$ for $908 \mathrm{~nm}$, and 9 for $1660 \mathrm{~nm}$ ). The mean error in the determination of the persistence length 

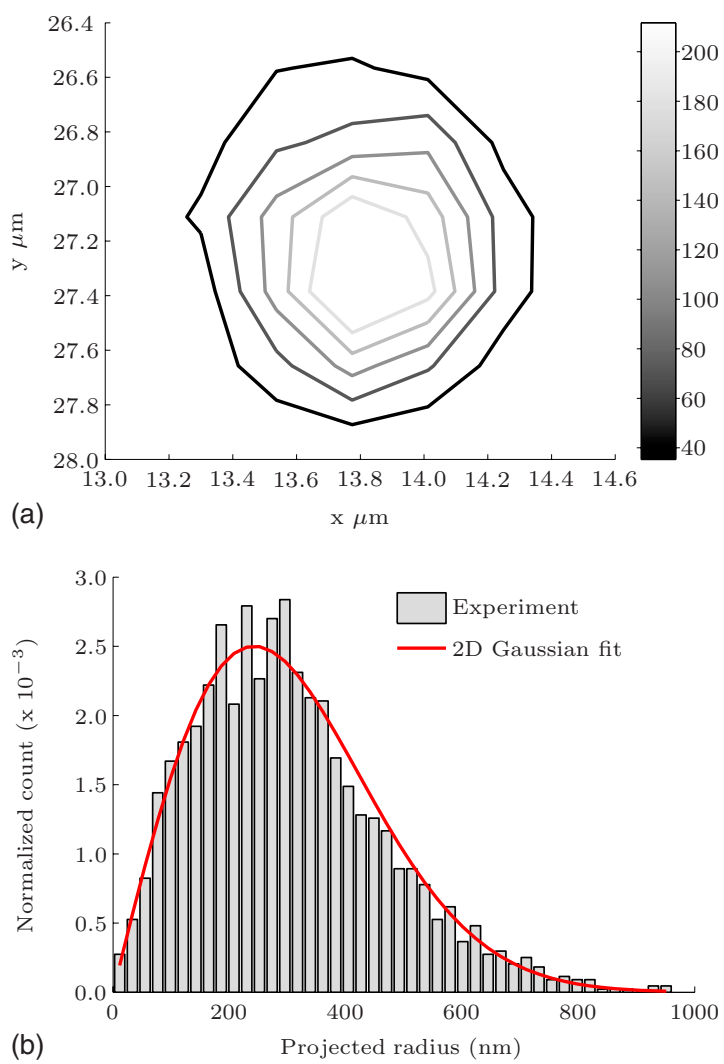

FIG. 7. (Color online) Results for a gold nanoparticle $(r=40 \mathrm{~nm})$ tethered to the surface using a $4882 \mathrm{bp}$ dsDNA molecule. (a) shows a 2D histogram of the positions of the nanoparticle during 2000 frames, while (b) shows the distribution of the projected radius of the particle positions. It can be fitted well by a Rayleigh distribution.

for a single molecule was $2 \mathrm{~nm}$. In Fig. 9 the results of the 45 molecules are combined. In Fig. 9(a) the full distribution of the measured persistence lengths is shown in a histogram. The persistence lengths we found had a mean value of $34.8 \mathrm{~nm}(\sigma=18.6 \mathrm{~nm})$ with standard error of $2.8 \mathrm{~nm}$. The mean persistence length corresponds to what other researchers have found under similar salt conditions (high $\mathrm{Na}^{+}$ concentration). ${ }^{4,40,41}$ In Fig. 9(b) the distribution of the mean square radii for the different dsDNA lengths is visualized in

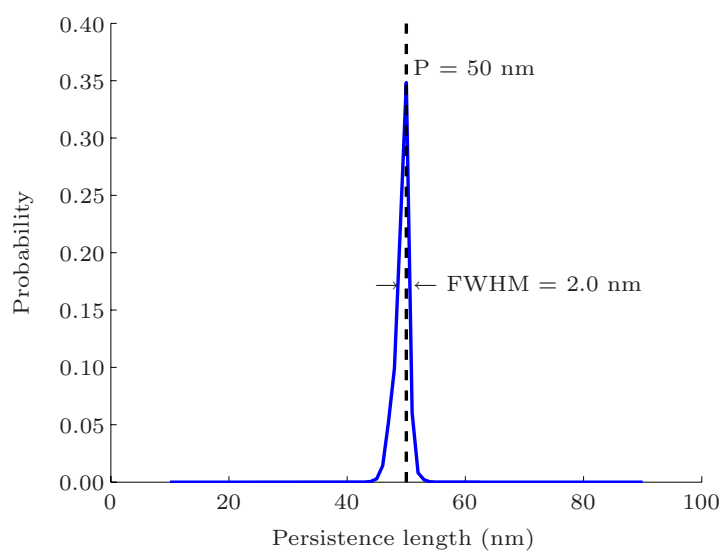

FIG. 8. (Color online) Results of two-sample KS test, comparing experimentally obtained particle positions to simulations using different persistence lengths. The figure shows that the best statistical agreement is with simulations using $50 \mathrm{~nm}$. The error in this determination is only $2.0 \mathrm{~nm}$.
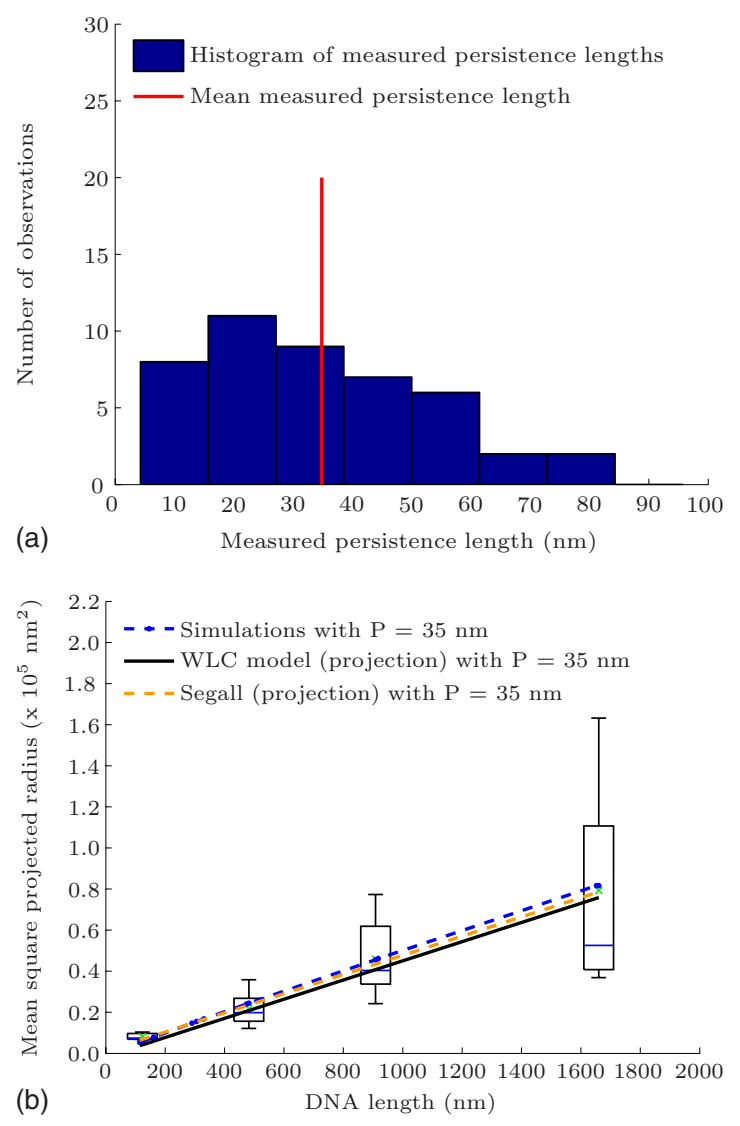

FIG. 9. (Color online) Results of the DNA experiments. (a) Distribution of the measured persistence length of 45 dsDNA molecules. (b) Mean square projected position as a function of DNA length, experiments (in box plots) compared to the simulations and theoretical models.

box plots. Overlayed on the box plots are the results of the simulations and the WLC model and influence of the volume exclusion, using $35 \mathrm{~nm}$ for the persistence length. Both models and the simulations fit the data very well: differences between these models and simulation are negligible compared to the measured variation between molecules.

\section{DISCUSSION}

In TPM experiments, the 2D projection of the position of the particle that is attached to the tether is imaged. The distribution of these positions should follow a Gaussian distribution, however, other researchers (e.g., Refs. 22 and 23) show that their experimental data cannot be described by such a curve. This can be explained by taking into account excluded volume effects due to the presence of a large particle at the end of the DNA. Segall et al. quantified this effect and defined a dimensionless excursion number to account for this effect. ${ }^{24}$ In other TPM experiments (e.g., Refs. 22 and 23 ), the bead radius is large compared to the persistence length and the contour length of the DNA; the excursion numbers are above one.

In our case the use of dark field microscopy permits much smaller particle radii, since the scattering of these particles is quite visible against the dark background. The excursion number posed by Segall et al. is well below the critical value of one and the volume exclusion effect is therefore negligible. This is confirmed by the fact that a fit of a $2 \mathrm{D}$ 


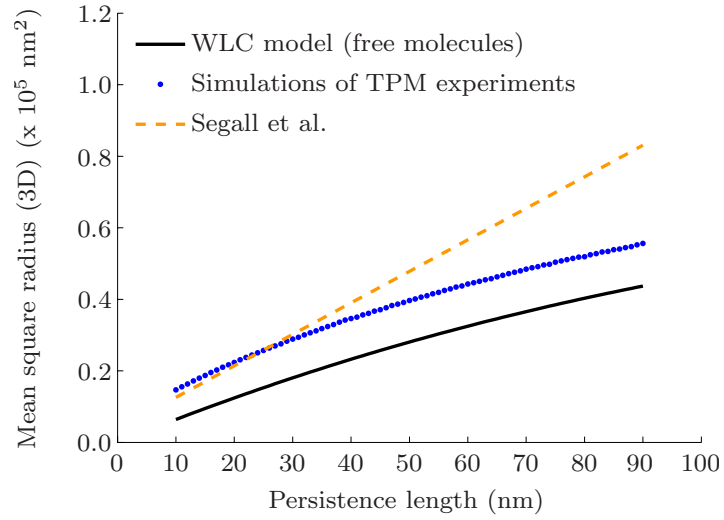

FIG. 10. (Color online) Second central moments of the (unaltered) WLC model, simulated molecules in TPM experiments and the model predicted by Segall et al. (Ref. 24) using 3D positional information for DNA with a contour length of $972 \mathrm{bp}(330 \mathrm{~nm})$.

Gaussian distribution to simulated [Fig. 5(c)] and experimental [Fig. 7(b)] data shows good agreement. This means that in our case there is no effective stretching force acting on the DNA and the DNA is in an unaltered state.

The excursion number was also used to predict a scaling of the central moments of the position distribution of freely jointed chains due to the volume exclusion effects. We have shown with our simulations that the behavior for WLCs differs from that model. If the tethered particle is small enough $\left(R_{p} \sim P\right)$, that scaling behavior does not even hold. However, this has not been verified experimentally, since the simulations and models do not differ more than the variation between single-molecule experiments if only the projected radius is collected [see Figs. 6(b) and 9(b)]. If height information of the nanoparticle is also taken into account, the differences between the models and simulation are larger (see Fig. 10).

Our measurements of the persistence length resulted in a mean value of $35 \mathrm{~nm}$ which is below the $50 \mathrm{~nm}$ value generally found in literature. However, over the years many researchers have determined the persistence length at different salt concentrations and temperatures resulting in values ranging between 30 and $80 \mathrm{~nm}^{40}$ Notably, methods wherein the rotational relaxation time of the DNA is measured even generate different values depending on the model that is used to calculate the persistence length from the experimental results. ${ }^{42}$ The general consensus is that at low salt concentrations, the self-interaction of the negatively charged DNA backbone results in a higher apparent persistence length. At higher salt concentrations this negative charge is screened by the positive ions in the buffer. Our measurements were done in a buffer with a relatively high salt concentration of 100 $\mathrm{mM}$ and compare well to other results in this concentration range. . $^{40,41}$

The large spread in the persistence lengths we measure resides in the fact that we have taken 45 individual, independent measurements: one for each molecule. Single-molecule measurements by AFM or EM, where the angle distribution or the end-to-end distance of the contour is used, are often analyzed by a sliding window approach over individual molecules, yielding a narrower persistence length distribution due to correlated evaluation. Furthermore, although it is common practice in TPM to discard any particle that exhibits significantly larger or smaller motion than is expected, ${ }^{19}$ we have taken into account all particles showing an isotropic position distribution to avoid biasing our measurements.

In contrast to other single-molecule methods such as AFM and EM where the environmental conditions are fixed, our method offers the possibility of measuring the influence of changing the temperature and buffer on the persistence length directly. Furthermore, it is possible to study the effect of interactions of the DNA tether with other molecules as for example proteins.

\section{CONCLUSIONS}

We have described a method for sensitively determining the persistence length of DNA. By determining the motion of a gold nanoparticle tethered to a substrate using a DNA molecule and comparing the obtained position distribution to simulations we are able to estimate the persistence length of single molecules with a precision of $2 \mathrm{~nm}$. The obtained projected position distributions have a Gaussian character, as expected from long polymers with negligible volume exclusion.

The measured persistence length of 45 single molecules with four different lengths in the same environmental conditions had a mean value of $35 \mathrm{~nm}$ (standard deviation of $18.6 \mathrm{~nm}$ ) with standard error of the mean $2.8 \mathrm{~nm}$. This complies with other results under the same salt conditions. Our method can also be used to directly measure the effect of environmental conditions on the persistence length, without additional external influences.

\section{ACKNOWLEDGMENTS}

The authors would like to thank Philip Nelson for generously providing his (MATHEMATICA) code for the Monte Carlo simulations and Keith Lidke for the CUDA code for the maximum likelihood estimation of the position of the Gaussian peaks. We would also like to thank Theo Odijk for numerous discussions. This work was partially supported by the BSIK program MicroNed, project 2F.

${ }^{1}$ O. Kratky and G. Porod, Recl. Trav. Chim. Pays-Bas 68, 1106 (1949).

${ }^{2}$ C. Bustamante, S. B. Smith, J. Liphardt, and D. Smith, Curr. Opin. Struct. Biol. 10, 279 (2000)

${ }^{3}$ G. Shivashankar, G. Stolovitzky, and A. Libchaber, Appl. Phys. Lett. 73, 291 (1998).

${ }^{4}$ M. D. Wang, H. Yin, R. Landick, J. Gelles, and S. M. Block, Biophys. J. 72, 1335 (1997).

${ }^{5}$ C. Bustamante, Z. Bryant, and S. B. Smith, Nature (London) 421, 423 (2003)

${ }^{6}$ S. B. Smith, L. Finzi, and C. Bustamante, Science 258, 1122 (1992).

${ }^{7}$ T. Strick, J.-F. Allemand, D. Bensimon, A. Bensimon, and V. Croquette, Science 271, 1835 (1996).

${ }^{8}$ C. Bouchiat, M. D. Wang, J.-F. Allemand, T. Strick, S. M. Block, and V. Croquette, Biophys. J. 76, 409 (1999).

${ }^{9}$ P. A. Wiggins, T. van der Heijden, F. Moreno-Herrero, A. Spakowitz, R. Philips, J. Windom, C. Dekker, and P. C. Nelson, Nat. Nanotechnol. 1, 137 (2006).

${ }^{10}$ J. Marek, E. Demjénová, Z. Tomori, J. Janácek, I. Zolotová, F. Valle, M. Favre, and G. Dietler, Cytometry, Part A 63A, 87 (2005).

${ }^{11}$ C. Rivetti, M. Guthold, and C. Bustamante, J. Mol. Biol. 264, 919 (1996).

${ }^{12}$ J. Bednar, P. Furrer, V. Katritch, A. Z. Stasiak, J. Dubochet, and A. 
Stasiak, J. Mol. Biol. 254, 579 (1995).

${ }^{13}$ D. Cherny and T. Jovin, J. Mol. Biol. 313, 295 (2001).

${ }^{14}$ K. M. Dohoney and J. Gelles, Nature (London) 409, 370 (2001).

${ }^{15}$ M. Capitanio, F. Vanzi, C. Broggio, R. Cicchi, D. Normanno, G. Romano, L. Sacconi, and F. Pavone, Microsc. Res. Tech. 65, 194 (2004).

${ }^{16}$ F. Vanzi, S. Vladimirov, C. R. Knudsen, Y. E. Goldman, and B. S. Cooperman, RNA 9, 1174 (2003).

${ }^{17}$ D. A. Schafer, J. Gelles, M. P. Sheetz, and R. Landick, Nature (London) 352, 444 (1991).

${ }^{18}$ B. van den Broek, F. Vanzi, D. Normanno, F. S. Pavone, and G. J. L. Wuite, Nucleic Acids Res. 34, 167 (2006).

${ }^{19}$ S. Blumberg, A. Gajraj, and M. W. Pennington, Biophys. J. 89, 1272 (2005).

${ }^{20}$ F. Vanzi, C. Broggio, L. Sacconi, and F. S. Pavone, Nucleic Acids Res. 34, 3409 (2006).

${ }^{21}$ H. R. C. Dietrich, F. H. de Groote, F. G. Wiertz, B. Rieger, H. A. Heering, I. T. Young, and Y. Garini, "DF-TPM: Tethered particle motion using darkfield microscopy," J. Nanophotonics (submitted).

${ }^{22}$ P. C. Nelson, C. Zurla, D. Brogioli, J. F. Beausang, L. Finzi, and D. Dunlap, J. Phys. Chem. B 110, 17260 (2006).

${ }^{23}$ N. Pouget, C. Dennis, C. Turlan, M. Grigoriev, M. Chandler, and L. Salome, Nucleic Acids Res. 32, e73 (2004).

${ }^{24}$ D. E. Segall, P. C. Nelson, and R. Phillips, Phys. Rev. Lett. 96, 088306 (2006).

${ }^{25}$ J. Yguerabide and E. E. Yguerabide, J. Cell Biochem. Suppl. 84(S37), 71 (2001).

${ }^{26}$ C. Bustamante, J. Marko, E. Siggia, and S. Smith, Science 265, 1599
(1994).

${ }^{27}$ Y. Shi, S. He, and J. E. Hearst, J. Chem. Phys. 105, 714 (1996).

${ }^{28}$ J. Samuel and S. Sinha, Phys. Rev. E 66, 050801 (2002).

${ }^{29}$ A. J. Spakowitz and Z.-G. Wang, Phys. Rev. E 72, 041802 (2005).

${ }^{30}$ S. Stepanow and G. M. Schütz, Europhys. Lett. 60, 546 (2002) (EPL).

${ }^{31}$ C. Hyeon, R. I. Dima, and D. Thirumalai, J. Chem. Phys. 125, 194905 (2006).

${ }^{32}$ M. Doi and S. Edwards, The Theory of Polymer Dynamics (Oxford University Press, New York, 1986).

${ }^{33}$ W. P. Wong and K. Halvorsen, Opt. Express 14, 12517 (2006).

${ }^{34}$ See EPAPS Document No. E-JCPSA6-130-026922 for the motion blur underestimation that occurs when imaging confined Brownian motion. For more information on EPAPS, see http://www.aip.org/pubservs/ epaps.html.

${ }^{35}$ R. J. Ober, S. Ram, and E. S. Ward, Biophys. J. 86, 1185 (2004).

${ }^{36}$ I. T. Young, J. Histochem. Cytochem. 25, 935 (1977).

${ }^{37}$ A. C. Cohen and B. J. Whitten, Parameter Estimation in Reliability and Life Span Models, Statistics: Textbooks and Monographs Vol. 96 (Dekker, New York, 1988).

${ }^{38}$ E. A. DiMarzio, J. Chem. Phys. 42, 2101 (1965).

${ }^{39}$ T. Odijk, personal communication (27 August 2008).

${ }^{40}$ P. Hagerman, Annu. Rev. Biophys. Bioeng. 17, 265 (1988).

${ }^{41}$ D. Porschke, Biophys. Chem. 40, 169 (1991).

${ }^{42}$ Y. Lu, B. Weers, and N. C. Stellwagen, Biopolymers 61, 261 (2002).

${ }^{43}$ Defined as $20 \log _{10}(\max (I) / \operatorname{std}(N)) . I$ and $N$ are the intensities and noise in the image. 